\title{
Entropy jumps for isotropic log-concave random vectors and spectral gap
}

\author{
Keith Ball \\ Institute of Mathematics, University of Warwick, \\ Coventry, CV4 7AL, UK \\ Email: kmb120205@googlemail.com \\ Van Hoang Nguyen \\ Institut de Mathématiques de Jussieu, UPMC, \\ 4 place Jussieu, 75252 Paris, France \\ Email: vanhoang@math.jussieu.fr
}

May 23, 2018

\begin{abstract}
We prove a quantitative dimension-free bound in the Shannon-Stam Entropy inequality for the convolution of two log-concave distributions in dimension $d$ in terms of the spectral gap of the density. The method relies on the analysis of the Fisher Information production, which is the second derivative of the Entropy along the (normalized) Heat semi-group. We also discuss consequences of our result in the study of the isotropic constant of log-concave distributions (slicing problem).
\end{abstract}

\section{Introduction}

Let $X$ be a random vector in $\mathbb{R}^{d}$ with density $f: \mathbb{R}^{d} \longrightarrow[0, \infty)$, a relation denoted by $X \sim f$. Its entropy is defined to be

$$
\operatorname{Ent}(X)=-\int_{\mathbb{R}^{d}} f \log f
$$

2010 Mathematics Subject Classification: 94A17.

Key words and phrases: Entropy, Fisher information, isotropic constant, isotropic log-concave random vector, spectral gap. 
provided $\int_{\mathbb{R}^{d}} f \log _{+} f<\infty$. We then say that $X$ has finite entropy.

We shall say that a random vector $X$ on $\mathbb{R}^{d}$, or a probability density $f$, is isotropic if it is centered and has a covariance matrix equal to the identity:

$$
\mathbb{E}[X]=\int_{\mathbb{R}^{d}} x f(x) d x=0
$$

and

$$
\mathbb{E}\left[X_{i} X_{j}\right]=\int_{\mathbb{R}^{d}} x_{i} x_{j} f(x) d x=\delta_{i, j}, \quad i, j=1 \ldots, d .
$$

This normalization can be realized by an affine transformation.

Among random vectors with a given covariance matrix, the corresponding Gaussian has the largest entropy. The gap between the entropy of a random vector $X$ and that of a Gaussian with same covariance matrix is a strong measure of how close $X$ is to being Gaussian. For instance, if $X$ has mean zero and is isotropic with density $f$, and if $G$ is a standard (normal) Gaussian vector with density $g$, then the Pinsker-Csiszár-Kullback inequality (see [17, 12] or [6]) implies that

$$
\frac{1}{2}\left(\int_{\mathbb{R}^{d}}|f-g|\right)^{2} \leq \operatorname{Ent}(G)-\operatorname{Ent}(X) .
$$

The celebrated Shannon-Stam inequality (see [22, 23]) says that if $X$ and $Y$ are independent identically distributed (iid in short) random vectors, then the normalized sum $(X+Y) / \sqrt{2}$ has entropy at least as large as that of $X$ and $Y$ :

$$
\operatorname{Ent}\left(\frac{X+Y}{\sqrt{2}}\right) \geq \operatorname{Ent}(X) \text {. }
$$

Moreover, this inequality is strict if $X$ is not itself a Gaussian random vector. A challenging problem is to quantify this phenomenon, i.e. for fixed covariance matrix (say the identity), bound $\operatorname{Ent}\left(\frac{X+Y}{\sqrt{2}}\right)-\operatorname{Ent}(X)$ from below by a non-negative (and positive outside zero) function of $\operatorname{Ent}(G)-\operatorname{Ent}(X)$. The first result in this direction was obtained by Carlen and Soffer [11] who proved, under technical assumptions, a non-explicit bound based on a compactness argument. Extra assumptions cannot be avoided if one aims at universal entropic estimates: it is easy to construct (by taking a carefully chosen double bumped Gaussian) a random variable $X$ for which, the convolution does not greatly modify the entropy, $\operatorname{Ent}\left(\frac{X+Y}{\sqrt{2}}\right) \simeq \operatorname{Ent}(X)$, but with $\operatorname{Ent}(X) \ll \operatorname{Ent}(G)$. A surprisingly neat result holds in the case where $X \in \mathbb{R}$ is a random variable with variance 1 and with a density $f$ that satisfies a Poincaré (or spectral gap) inequality in the sense that for some positive $c$ and any smooth function $s$ with $\int_{\mathbb{R}} f s=0$

$$
c \int_{\mathbb{R}} f s^{2} \leq \int_{\mathbb{R}} f\left(s^{\prime}\right)^{2} .
$$

Indeed, we then have

$$
\operatorname{Ent}\left(\frac{X+Y}{\sqrt{2}}\right)-\operatorname{Ent}(X) \geq \frac{c}{2+2 c}(\operatorname{Ent}(G)-\operatorname{Ent}(X))
$$


for $Y$ an independent copy of $X$. This result was proved by Ball, Barthe and Naor in [3] using a variational formula for the Fisher information of a marginal density and spectral analysis to get an information jump in the presence of a spectral gap (see [3, Theorem $2]$ ) and then using a relation between the Fisher information and entropy provided by the adjoint Ornstein-Uhlenbeck semigroup. In the paper [5], Barron and Johnson obtained a result similar to (1.1) under the same hypothesis, but their method is different (at least in details) to that of [3]. In their paper, Barron and Johnson give an upper bound for the $L^{2}$ distance of the score function of $(X+Y) / \sqrt{2}$ to the space of additive functions of $X$ and $Y$, using $L^{2}$-orthogonal projections; they eventually use the Poincaré inequality to get the result (see [5, Proposition 2.1 and 3.1] for details).

The aim of the present paper is to investigate similar results for random vectors, and incidentally to give a new approach to (1.1). A random vector $X \in \mathbb{R}^{d}$ with density $f$ is said to satisfy a Poincaré or spectral gap inequality with constant $c>0$ if for any smooth function $s$ with $\int_{\mathbb{R}^{d}} f s=0$

$$
c \int_{\mathbb{R}^{d}} f s^{2} \leq \int_{\mathbb{R}^{d}} f|\nabla s|^{2} .
$$

That largest constant $c$ in this inequality is indeed the spectral gap for the operator $-L$ on $L^{2}(f)$ where $L s:=\Delta s-\nabla(\log (f) \cdot \nabla s$ for suitable functions; the Poincaré constant $c_{\mathrm{p}}(f)$ refers rather to the inverse of the spectral gap, i.e. to the smallest constant in the inequality

$$
\int_{\mathbb{R}^{d}} f s^{2} \leq \mathrm{c}_{\mathrm{p}}(f) \int_{\mathbb{R}^{d}} f|\nabla s|^{2} .
$$

A simplistic adaptation of the argument used [3] in higher dimensions leads to an inequality of the form (1.1) for random vectors but with an extra dependance in $d$, the dimension. In the present paper we prove the result without the extra dependence for $\log$-concave random vectors, i.e. those having a density $f$ such that $-\log (f)$ is convex on $\mathbb{R}^{n}$. It is well known that such random vectors have finite entropy and have a positive spectral gap (see below). This family is central in many high-dimensional problems.

Theorem 1. Let $X$ be an isotropic log-concave random vector in $\mathbb{R}^{d}$. Assume its density $f$ satisfies a Poincaré inequality (1.2) with constant $c>0$. Then, if $Y$ is an independent copy of $X$, we have

$$
\operatorname{Ent}\left(\frac{X+Y}{\sqrt{2}}\right)-\operatorname{Ent}(X) \geq \frac{c}{4(1+c)}(\operatorname{Ent}(G)-\operatorname{Ent}(X)) .
$$

Since $c \leq 1$ when $f$ is isotropic, the constant $\frac{c}{4(1+c)}$ may be replaced by $\frac{c}{8}$. The $\log$ concavity assumption will be crucial on the proof of inequality (3.2) below, we do not know whether it holds without this assumption.

As mentioned above, we need to develop a method different to the one of [3]. Our alternative approach relies on the study of second derivatives of the entropy along the heat semi-group (or rather along the Ornstein-Uhlenbeck semi-group). Note that it also gives an alternative proof of the one dimensional case (1.1), up to a numerical (nonessential) constant. 
Let us make some general comments on log-concave random vectors. If $X$ and $Y$ are i.i.d random vectors with density $f$, the normalized sum $\frac{X+Y}{\sqrt{2}}$ has density

$$
u \longrightarrow \int_{\mathbb{R}^{d}} f\left(\frac{u+v}{\sqrt{2}}\right) f\left(\frac{u-v}{\sqrt{2}}\right) d v
$$

which is a marginal of the joint density on $\mathbb{R}^{2 d}$ of the pair $(X, Y)$. It is a consequence of the Brunn-Minkowski inequality (in its functional form due to Prékopa [21]) that log-concave random vectors have log-concave marginals and hence that if $X$ and $Y$ are log-concave, then so is $\frac{X+Y}{\sqrt{2}}$. It is also well known that a log-concave density verifies a Poincaré inequality for some constant $c>0$. It was proven by Kannan, Lovasz and Simonovitz [18] and independently by Bobkov [7] that if $X$ is an isotropic log-concave random vector, then it

satifies a Poincaré inequality (1.2) with consant $\frac{C}{n}$ for some numerical constant $C$ (thus independent of $n$ and $X$ ). Actually, slightly better depedence in $n$ is known. The KannanLovasz-Simonovitz (KLS) open conjecture states that there exists a universal constant $c>0$ such that for every $n$, every every isotropic log-concave random vector in $\mathbb{R}^{n}$ verifies a Poincaré inequality with constant $c$. It was noticed some time ago by the first named author, as part of a general program of understanding information theory (and entropy) in the context of convexity in high dimensions, that using (1.4) we can prove that the KLS conjecture implies the celebrated hyperplane (or slicing) conjecture. We shall return to this in the last section.

The organization of the paper is as follows. In the next section, we recall some standard facts about the Ornstein-Uhlenbeck semi-group and about the first derivative of entropy (Fisher information) and the second derivative (information production). Next we investigate how information production behaves under convolution and state a general inequality relating information production of a random vector to the information of a marginal. The subsequent section contains the proof of Theorem 1. The final section discusses the connections between entropy jump and the isotropic constant of log-concave distribution.

\section{Classical results on Ornstein-Uhlenbeck semi-group and Fisher information}

For any random vector $X$ with smooth enough density $f$-we require that $\sqrt{f} \in H^{1}\left(\mathbb{R}^{n}\right)$, but later the density will have even smoother behavior- its Fisher information is defined by

$$
J(X):=J(f):=\int_{\mathbb{R}^{d}} \frac{|\nabla f|^{2}}{f} .
$$

Among random vectors with given covariance matrix, the Gaussian has the smallest Fisher information, as shown by the following straightforward computation: if $X$ is an isotropic mean-zero log-concave random with density $f$, and $G$ is a standard Gaussian 
with density $g: x \rightarrow(1 / \sqrt{2 \pi})^{d} e^{-|x|^{2} / 2}$, then

$$
J(G)=\int_{\mathbb{R}^{d}} \frac{|\nabla g|^{2}}{g}=\frac{1}{\sqrt{2 \pi}^{d}} \int_{\mathbb{R}^{d}} \sum_{i=1}^{d} x_{i}^{2} e^{-|x|^{2} / 2} d x=d .
$$

and, by integration by parts,

$$
0 \leq \int_{\mathbb{R}^{d}}\left|\frac{\nabla f}{f}+x\right|^{2} f(x) d x=J(X)-2 \int_{\mathbb{R}^{d}} \operatorname{div}(x) f+d=J(X)-J(G) .
$$

The Fisher information appears as the derivative of the entropy along the OrnsteinUhlenbeck semi-group, a property central in the works by Bakry and Émery [1] and also in Barron's work [4] on the convergence of entropy in the central limit theorem. The Ornstein-Uhlenbeck semi-group can be constructed in several (equivalent) ways and we choose the following. If $X$ is a random vector with density $f$ and $G$ is a standard Gaussian, independent of $X$, we consider the random vector $X_{t}=e^{-t} X+\sqrt{1-e^{-2 t}} G$, whose law is the Ornstein-Uhlenbeck evolute at time $t$ of the law of $X$. More precisely, the density $f_{t}$ of $X_{t}$ is the solution of the Fokker-Planck equation with generator $L$ :

$$
f_{0}=f ; \quad \frac{\partial}{\partial t} f_{t}(x)=L\left(f_{t}\right)(x):=\Delta_{x} f_{t}+\operatorname{div}_{x}\left(x f_{t}\right)
$$

for all $t>0$ and $x \in \mathbb{R}^{n}$.

It is indeed well known, that starting with a (continuous, say) density $f$, the density $f_{t}$ is, for $t>0$, strictly positive, $C^{\infty}$-smooth on $\mathbb{R}^{n}$ and that $f_{t}$ and its derivatives decay exponentially fast to zero at $\infty$; in particular $f_{t}$ has a finite Fisher information and it is readily checked that

$$
\begin{aligned}
\frac{\partial}{\partial_{t}} \operatorname{Ent}\left(f_{t}\right) & =-\int_{\mathbb{R}^{d}}\left(L f_{t}\right) \log \left(f_{t}\right)=-\int_{\mathbb{R}^{d}} f_{t} \Delta \log \left(f_{t}\right)-d \int_{\mathbb{R}^{d}} f_{t} \\
& =J\left(f_{t}\right)-d .
\end{aligned}
$$

Hence, we have the classical expression of the entropy gap as the integral of the information gap

$$
\operatorname{Ent}(G)-\operatorname{Ent}(X)=\int_{0}^{\infty}\left(J\left(f_{t}\right)-d\right) d t
$$

We refer to Carlen and Soffer [11] for details and precise justifications.

Let us mention for further reference some other nice stability properties of the OrnsteinUhlenbeck semi-group. It can only improve the spectral gap: if $X \sim f$ is isotropic and satisfies a Poincaré inequality (1.2) with constant $c>0$ (actually, $c \in] 0,1]$ ), then $f_{t}$ satisfies a Poincaré inequality with the same constant $c>0$. This follows easily from Fubini's theorem, Hölder's inequality and the fact that the Gaussian density has spectral gap of size 1 (see [3]). Next, it is again a consequence of Prékopa's theorem that if $X$ (or $f$ ) is log-concave, then so is $X_{t}\left(\right.$ or $\left.f_{t}\right)$. Finally, it is also classical that the operation 
of taking marginals commutes with the Ornstein-Uhlenbeck semi-group in the following sense. Let $X$ and $Y$ be two independent random vectors and $X_{t}=e^{-t} X+\sqrt{1-e^{-2 t}} G_{1}$ and $Y_{t}=e^{-t} Y+\sqrt{1-e^{-2 t}} G_{2}$ their independent evolutes along the Ornstein-Uhlenbeck semi-group, where each $G_{i}(i=1,2)$ is a standard Gaussian vector independent of all the other vectors. Then

$$
\frac{X_{t}+Y_{t}}{\sqrt{2}}=e^{-t} \frac{X+Y}{\sqrt{2}}+\sqrt{1-e^{-2 t}} G
$$

where $G=\frac{G_{1}+G_{2}}{\sqrt{2}}$ is a standard Gaussian vector.

Throughout the rest of the section, $X$ will be an isotropic log-concave random vector with mean zero, and density $f$. The density $f_{t}$ of $X_{t}=e^{-t} X+\sqrt{1-e^{-2 t}} G$, the evolute of $X$ along the Ornstein-Uhlenbeck semi-group satisfies (2.1) and takes the form $f_{t}=e^{-\varphi_{t}}$ with $\varphi_{t}:=\log \left(f_{t}\right)$ convex on $\mathbb{R}^{d}$. Its Fisher information will be denoted by

$$
J(t):=J\left(f_{t}\right)=\int_{\mathbb{R}^{d}} \frac{\left|\nabla f_{t}\right|^{2}}{f_{t}}=-\int_{\mathbb{R}^{d}} f_{t} \Delta \log f_{t}=\operatorname{Tr} \int_{\mathbb{R}^{d}} f_{t} \operatorname{Hess} \varphi_{t} .
$$

We will work with the derivative $\partial_{t} J(t)$ of the Fisher information along the OrnsteinUhlenbeck semi-group. The following result is classical in the context of Bakry-Emery's $\Gamma_{2}$ calculus, although it is not usually written in this form which for us will prove useful. We include a proof for completeness.

Lemma 2. With the previous notation we have

$$
\partial_{t} J(t)=2 J(t)-2 \operatorname{Tr} \int_{\mathbb{R}^{d}} f_{t}\left(\operatorname{Hess} \varphi_{t}\right)^{2} .
$$

Proof. Denoting $\partial_{j}$ the partial derivative (in space) with respect to $x_{j}$ we have

$$
\begin{aligned}
\partial_{t} J(t)= & \sum_{i=1}^{d} \int_{\mathbb{R}^{d}} 2 \frac{\partial_{i} f_{t}}{f_{t}} \partial_{i} \partial_{t} f_{t}-\int_{\mathbb{R}^{d}}\left(\frac{\partial_{i} f_{t}}{f_{t}}\right)^{2} \partial_{t} f_{t} \\
= & \sum_{i=1}^{d} \int_{\mathbb{R}^{d}}-2 \partial_{i} \varphi_{t} \partial_{i}\left[\sum_{j=1}^{d} \partial_{j}\left(\left(-\partial_{j} \varphi_{t}+x_{j}\right) f_{t}\right)\right] \\
& -\int_{\mathbb{R}^{d}} \sum_{j=1}^{d} \partial_{j}\left(\left(-\partial_{j} \varphi_{t}+x_{j}\right) f_{t}\right)\left(\partial_{i} \varphi_{t}\right)^{2} .
\end{aligned}
$$

where we used that $f_{t}$ follows (2.1) and $L g=\sum_{j} \partial_{j}\left(\partial_{j} g+x_{j} g\right)$. Let $A$ and $B$ be the first and the second terms in the above sum, respectively. Then, we have, by integration by parts and (2.4),

$$
\begin{aligned}
A & =2 \sum_{i, j=1}^{d} \int_{\mathbb{R}^{d}}\left(\partial_{i j} \varphi_{t}\right) \partial_{i}\left(\left(-\partial_{j} \varphi_{t}+x_{j}\right) f_{t}\right) \\
& =2 J(t)-2 \operatorname{Tr} \int_{\mathbb{R}^{d}} f_{t}\left(\operatorname{Hess} \varphi_{t}\right)^{2}+2 \sum_{i, j=1}^{d} \int_{\mathbb{R}^{d}} f_{t}\left(\partial_{i j} \varphi_{t}\right)\left(\partial_{i} \varphi_{t}\right)\left(\partial_{j} \varphi_{t}-x_{j}\right)
\end{aligned}
$$


and

$$
\begin{aligned}
B & =-\sum_{i=1}^{d} \int_{\mathbb{R}^{d}} \sum_{j=1}^{d} \partial_{j}\left(f_{t}\left(-\partial_{j} \varphi_{t}+x_{j}\right)\right)\left(\partial_{i} \varphi_{t}\right)^{2} \\
& =2 \sum_{i, j=1}^{d} \int_{\mathbb{R}^{d}} f_{t}\left(-\partial_{j} \varphi_{t}+x_{j}\right)\left(\partial_{i} \varphi_{t}\right)\left(\partial_{i j} \varphi_{t}\right) \\
& =-2 \sum_{i, j=1}^{d} \int_{\mathbb{R}^{d}} f_{t}\left(\partial_{i j} \varphi_{t}\right)\left(\partial_{i} \varphi_{t}\right)\left(\partial_{j} \varphi_{t}-x_{j}\right) .
\end{aligned}
$$

Taking the sum of $A$ and $B$, one gets the result of this lemma.

Note that the formula in the previous lemma can be rewritten in the following equivalent form, which is more standard:

$$
\begin{aligned}
\partial_{t}(J(t)-d) & =-2(J(t)-d)-2 \operatorname{Tr} \int_{\mathbb{R}^{d}} f_{t}\left(\operatorname{Hess} \varphi_{t}-\mathrm{Id}\right)^{2} \\
& \leq-2(J(t)-d)
\end{aligned}
$$

The next lemma will allow us to control the tails of the entropy production.

Lemma 3. With the previous notation we have

$$
\operatorname{Ent}(G)-\operatorname{Ent}(X) \leq 2 \int_{0}^{\infty} e^{-2 t}(J(t)-d) d t
$$

Proof. Integration of inequality (2.6) leads to the following classical Gaussian Log-Sobolev inequality

$$
J(t)-d \geq 2\left(\operatorname{Ent}(G)-\operatorname{Ent}\left(X_{t}\right)\right) \forall t>0 .
$$

By integration by parts, we get

$$
\int_{0}^{\infty} e^{-2 t}(J(t)-d) d t \geq \operatorname{Ent}(G)-\operatorname{Ent}(X)-\int_{0}^{\infty} e^{-2 t}(J(t)-d) d t
$$

or equivalentely

$$
\operatorname{Ent}(G)-\operatorname{Ent}(X) \leq 2 \int_{0}^{\infty} e^{-2 t}(J(t)-d) d t
$$

\section{A result for the information production of marginals}

As we saw in (2.5), the information production $\partial_{t} J(t)$ along the Ornstein-Uhlenbeck semigroup is given by quantities of the form

$$
\operatorname{Tr} \int(\operatorname{Hess} \log f)^{2} f .
$$


For our argument, we need to analyze how such quantities can be estimated for marginal densities. Assume $Z$ is a random vector with density $\omega: \mathbb{R}^{N} \rightarrow \mathbb{R}_{+}(N \geq 1)$ and consider the projection $P_{E} Z$ of $Z$ onto a subspace $E \subset \mathbb{R}^{N}$. It has a density on $E \simeq \mathbb{R}^{\operatorname{dim}(E)}$ which we denote by $h$. A useful observation due to Carlen [10] for Fisher information is that

$$
J(h) \leq \int \frac{\left|P_{E} \nabla \omega\right|^{2}}{\omega} .
$$

The next result provides an analogue for information production. However, we are able to establish it only in the case of log-concave densities: here is where the restriction in our main theorem comes from. Using it, we can then state the central inequality that will be used in the proof of the main theorem.

Lemma 4. Let $N \geq 1$ and $\omega=e^{-\phi}: \mathbb{R}^{N} \rightarrow \mathbb{R}_{+}$be a smooth positive function. Given a subspace $E \subset \mathbb{R}^{N}$ define the marginal function on $E$ by

$$
\forall x \in E, \quad h(x):=e^{-\psi(x)}:=\int_{E^{\perp}} \omega(x+y) d y=\int_{E^{\perp}} e^{-\phi(x+y)} d y .
$$

Denote by $P_{E}$ the orthogonal projection onto $E$. Then, for every $x \in E$ we have,

$$
h(x) \operatorname{Hess} \psi(x) \leq \int_{E^{\perp}} \omega(x+y) P_{E} \operatorname{Hess} \phi(x+y) P_{E} d y
$$

in the operator sense (for symmetric operators on $E$ ) and if Hess $\psi(x) \geq 0$, then

$$
\operatorname{Tr}\left[(\operatorname{Hess} \psi(x))^{2} h(x)\right] \leq \int_{E^{\perp}} \operatorname{Tr}\left[\left(P_{E} \operatorname{Hess} \phi(x+y) P_{E}\right)^{2}\right] \omega(x+y) d y .
$$

Therefore, if Hess $\psi \geq 0$ we have

$$
\int_{E} \operatorname{Tr}\left[(\operatorname{Hess} \log h)^{2}\right] h \leq \int_{\mathbb{R}^{N}} \operatorname{Tr}\left[\left(P_{E}(\text { Hess } \log \omega) P_{E}\right)^{2}\right] \omega .
$$

Proof. We start with the observation that for $x \in E$,

$$
\frac{\nabla h(x) \otimes \nabla h(x)}{h(x)} \leq \int_{E^{\perp}} \frac{P_{E} \nabla \omega(x+y) \otimes P_{E} \nabla \omega(x+y)}{\omega(x+y)} d y
$$

in the symmetric operator sense (on $E$ ). Indeed, we have

$$
\nabla h(x)=\int_{E^{\perp}} P_{E} \nabla \omega(x+y) d y
$$

and for any $v \in E$ we have, using the Cauchy-Schwartz inequality

$$
(\nabla h(x), v)^{2} \leq \int_{E^{\perp}} \frac{(\nabla \omega(x+y), v)^{2}}{\omega(x+y)} d y \int_{E^{\perp}} \omega(x+y) d y
$$


as claimed. Next, observe that

$$
\operatorname{Hess} h(x)=\int_{E_{\perp}} P_{E} \operatorname{Hess} \omega(x+y) P_{E} d y
$$

and

$$
h(x) \operatorname{Hess} \psi(x)=h(x) \operatorname{Hess}(-\log h)(x)=\frac{\nabla h(x) \otimes \nabla h(x)}{h(x)}-\operatorname{Hess} h(x) .
$$

Thus (3.3) leads to the inequality

$$
\begin{aligned}
h(x) \operatorname{Hess} \psi(x) & \leq \int_{E^{\perp}}\left(\frac{P_{E} \nabla \omega(x+y) \otimes P_{E} \nabla \omega(x+y)}{\omega(x+y)}-P_{E} \operatorname{Hess} \omega(x+y) P_{E}\right) d y \\
& =\int_{E^{\perp}} \omega(x+y) P_{E} \operatorname{Hess} \phi(x+y) P_{E} d y
\end{aligned}
$$

in the operator sense on $E$, as wanted.

Using that that for symmetric operators $A \geq B \Rightarrow \operatorname{Tr}(A H) \geq \operatorname{Tr}(B H)$ whenever $H \geq 0$, we deduce that, when Hess $\psi(x) \geq 0$,

$$
\operatorname{Tr}\left[(\operatorname{Hess} \psi(x))^{2} h(x)\right] \leq \int_{E^{\perp}} \operatorname{Tr}\left[P_{E} \operatorname{Hess} \phi(x+y) P_{E} \operatorname{Hess} \psi(x)\right] \omega(x+y) d y .
$$

By the Cauchy-Schwartz inequality (in vectorial form, for the Hilbert-Schmidt scalar product) we then have

$$
\begin{aligned}
\operatorname{Tr}\left[(\operatorname{Hess} \psi(x))^{2} h(x)\right] \leq & \sqrt{\int_{E^{\perp}} \operatorname{Tr}\left[\left(P_{E} \operatorname{Hess} \phi(x+y) P_{E}\right)^{2}\right] \omega(x+y) d y} \\
& \times \sqrt{\int_{E^{\perp}} \operatorname{Tr}\left[(\operatorname{Hess} \psi(x))^{2}\right] \omega(x+y) d y .}
\end{aligned}
$$

Noting that the second integral equals $\operatorname{Tr}\left[(\operatorname{Hess} \psi(x))^{2} h(x)\right]$, we arrive to inequality (3.1). Integration over $E$ then gives (3.2).

For our argument, we will need the following useful observation.

Theorem 5. Let $X$ be a log-concave random vector in $\mathbb{R}^{d}$ with smooth density $f=e^{-\varphi}$ where $\varphi$ is a convex function on $\mathbb{R}^{d}$, and let $Y$ be an independent copy of $X$. Denote by $h=e^{-\psi}$ the density on $\mathbb{R}^{d}$ of the random vector $\frac{X+Y}{\sqrt{2}}$ and put

$$
K=\operatorname{Tr}\left[\int_{\mathbb{R}^{d}}(\operatorname{Hess} \varphi)^{2} f\right], \quad K_{2}=\operatorname{Tr}\left[\int_{\mathbb{R}^{d}}(\operatorname{Hess} \psi)^{2} h\right],
$$

and

$$
M=\operatorname{Tr}\left[\left(\int_{\mathbb{R}^{d}}(\operatorname{Hess} \varphi) f\right)^{2}\right] .
$$

Then, we have

$$
K_{2} \leq \frac{K+M}{2}
$$


Proof. As mentioned earlier, we know by Prékopa's theorem that $h$ is log-concave, i.e. Hess $\psi \geq 0$.

We denote $\omega(x, y)=f(x) f(y)$ the density of $(X, Y)$ on $\mathbb{R}^{d} \times \mathbb{R}^{d}=\mathbb{R}^{2 d}$. For $i=1, \ldots d$ we set $e_{i}=\left(0, \ldots, \frac{1}{\sqrt{2}}, 0, \ldots, 0, \frac{1}{\sqrt{2}}, 0, \ldots\right)$ where the $i$-th and $(d+i)$-th coordinates are equal to $\frac{1}{\sqrt{2}}$ and the others are zero. Let $E$ be the vector subspace of $\mathbb{R}^{2 d}$ spanned by the orthogonal family $\left\{e_{1}, \cdots, e_{d}\right\}$.We can assume that the density $h=e^{-\psi}$ of the random vector $\frac{X+Y}{\sqrt{2}}$ is defined on $E$ by identification of $\mathbb{R}^{d}$ and $E$ through the orthonormal basis $\left\{e_{i}\right\}$ of $E$. Then the Lemma 4 gives

$$
\begin{aligned}
K_{2} & \leq \int_{\mathbb{R}^{2 d}} \omega(x, y) \operatorname{Tr}\left[\left[\left(\operatorname{Hess}(-\log \omega)(x, y) e_{i}, e_{j}\right)\right]_{i, j}^{2}\right] d x d y \\
& =\int_{\mathbb{R}^{2 d}} f(x) f(y) \sum_{i, j=1}^{d}\left(\operatorname{Hess}(-\log \omega)(x, y) e_{i}, e_{j}\right)^{2} d x d y .
\end{aligned}
$$

Direct computation gives

$$
\left(\operatorname{Hess}(-\log \omega)(x, y) e_{i}, e_{j}\right)^{2}=\frac{1}{4}\left(\partial_{j i} \varphi(x)+\partial_{j i} \varphi(y)\right)^{2}
$$

and hence

$$
\begin{aligned}
K_{2} & \leq \sum_{i, j=1}^{d} \frac{1}{4} \int_{\mathbb{R}^{2 d}} f(x) f(y)\left(\left(\partial_{j i} \varphi(x)\right)^{2}+2 \partial_{j i} \varphi(x) \partial_{j i} \varphi(y)+\left(\partial_{j i} \varphi(y)\right)^{2}\right) d x d y \\
& =\frac{1}{2} \sum_{i, j=1}^{d} \int_{\mathbb{R}^{d}} f\left(\partial_{j i} \varphi\right)^{2}+\frac{1}{2} \sum_{i, j=1}^{d}\left(\int_{\mathbb{R}^{d}} f \partial_{j i} \varphi\right)^{2} \\
& =\frac{1}{2}(K+M) .
\end{aligned}
$$

\section{Proof of Theorem 1.1}

We go back to the situation and the notation of Section $\$ 2, X$ is an isotropic log-concave random vector with mean zero, and density $f$, and $X_{t}$ is its evolute along the OrnsteinUhlenbeck semi-group. The (log-concave) density of $X_{t}$ is denoted by $f_{t}=e^{-\varphi_{t}}$ and we set

$$
J(t):=J\left(X_{t}\right)=\operatorname{Tr} \int_{\mathbb{R}^{d}} f_{t} \operatorname{Hess} \varphi_{t}
$$

and

$$
K(t):=\operatorname{Tr} \int_{\mathbb{R}^{d}} f_{t}\left(\operatorname{Hess} \varphi_{t}\right)^{2}=-\frac{1}{2} e^{2 t} \partial_{t}\left(e^{-2 t} J(t)\right)
$$

where we used Lemma 2 for the last equality. 
We now consider $Z_{t}$, the Ornstein-Uhlenbeck evolute of $\frac{X+Y}{\sqrt{2}}$ where $Y$ is an independent copy of $X$. As mentioned earlier (2.3),$Z_{t}=\frac{X_{t}+Y_{t}}{\sqrt{2}}$ where $Y_{t}$ is an Ornstein-Uhlenbeck evolute of $Y$ independent of $X_{t}$. Denote by $h_{t}=e^{-\psi_{t}}$ the smooth (log-concave) density of $Z_{t}$ and set accordingly

$$
J_{2}(t):=J\left(Z_{t}\right)=\operatorname{Tr} \int_{\mathbb{R}^{d}} h_{t} \operatorname{Hess} \psi_{t}
$$

and

$$
K_{2}(t):=\operatorname{Tr} \int_{\mathbb{R}^{d}} h_{t}\left(\operatorname{Hess} \psi_{t}\right)^{2}=-\frac{1}{2} e^{2 t} \partial_{t}\left(e^{-2 t} J_{2}(t)\right)
$$

Theorem 5 applied to $X_{t}$ and $Z_{t}=\frac{X_{t}+Y_{t}}{\sqrt{2}}$ then gives that

$$
K_{2}(t) \leq \frac{K(t)+M(t)}{2}=K(t)-\frac{K(t)-M(t)}{2}
$$

where,

$$
M(t):=\operatorname{Tr}\left[\left(\int_{\mathbb{R}^{d}}\left(\operatorname{Hess} \varphi_{t}\right) f_{t}\right)^{2}\right]
$$

This can be rewritten as

$$
\partial_{t}\left(e^{-2 t}\left(J_{2}(t)-J(t)\right)\right) \geq e^{-2 t}(K(t)-M(t))
$$

We next claim that

$$
K(t)-M(t) \geq \frac{c}{1+c}(K(t)-J(t))
$$

To prove this, remember, as recalled in Section §2, that $f_{t}$ verifies Poincaré's inequality with the same (or better) constant $c$ as $f$. We apply the Poincaré inequality (1.2) to the density $f_{t}=e^{-\varphi_{t}}$ and to the functions

$$
s_{i}(x)=\partial_{i} \varphi_{t}(x)-\sum_{j=1}^{d} x_{j} \int_{\mathbb{R}^{d}}\left(\partial_{i j} \varphi_{t}\right) f_{t}
$$

which verify that $\int s_{i} f_{t}=0$, for $i=1, \ldots, d$. After summing the inequalities $\int_{\mathbb{R}^{d}}\left|\nabla s_{i}\right|^{2} f_{t} \geq$ $c \int_{\mathbb{R}^{d}} s_{i}^{2} f_{t}$ we find

$$
\operatorname{Tr} \int_{\mathbb{R}^{d}} f_{t}\left(\operatorname{Hess} \varphi_{t}\right)^{2}-\operatorname{Tr}\left(\int_{\mathbb{R}^{d}} f_{t} \operatorname{Hess} \varphi_{t}\right)^{2} \geq c\left(\operatorname{Tr}\left(\int_{\mathbb{R}^{d}} f_{t} \operatorname{Hess} \varphi_{t}\right)^{2}-\operatorname{Tr} \int_{\mathbb{R}^{d}} f_{t} \operatorname{Hess} \varphi_{t}\right) .
$$

This rewrites as $K(t)-M(t) \geq c(M(t)-J(t))$, which is equivalent to the desired inequality (4.2).

Substituting inequality (4.2) in (4.1), we find

$$
\partial_{t}\left(e^{-2 t}\left(J_{2}(t)-J(t)\right)\right) \geq \frac{c}{1+c} e^{-2 t}(K(t)-J(t)) .
$$


Integrating this inequality from $t$ to $\infty$, we obtain

$$
J(t)-J_{2}(t) \geq \frac{c}{1+c} e^{2 t} \int_{t}^{\infty} e^{-2 s}(K(s)-J(s)) d s .
$$

Hence, using (2.2),

$$
\begin{aligned}
\operatorname{Ent}\left(\frac{X+Y}{\sqrt{2}}\right)-\operatorname{Ent}(X) & =\int_{0}^{\infty}\left(J(t)-J_{2}(t)\right) d t \\
& \geq \frac{c}{1+c} \int_{0}^{\infty} e^{2 t} \int_{t}^{\infty} e^{-2 s}(K(s)-J(s)) d s d t \\
& =\frac{c}{2(1+c)} \int_{0}^{\infty}\left(1-e^{-2 t}\right)(K(t)-J(t)) d t \\
& =\frac{c}{2(1+c)} \int_{0}^{\infty}\left(1-e^{-2 t}\right)\left(-\frac{1}{2} \partial_{t}(J(t)-d)\right) d t \\
& =\frac{c}{2(1+c)} \int_{0}^{\infty} e^{-2 t}(J(t)-d) d t
\end{aligned}
$$

Applying Lemma 3 we get

$$
\operatorname{Ent}\left(\frac{X+Y}{\sqrt{2}}\right)-\operatorname{Ent}(X) \geq \frac{c}{4(1+c)}(\operatorname{Ent}(G)-\operatorname{Ent}(X))
$$

This ends the proof of Theorem 1.

\section{Links with the Isotropic constant}

The isotropic constant of an isotropic log-concave random vector $X \sim f$ on $\mathbb{R}^{d}$ is defined by

$$
L_{X}:=L_{f}:=f(0)^{1 / d}
$$

This quantity appears in several high-dimensional problems and a challenging open problem in asymptotic convex geometry raised by Bourgain and known as the Slicing or Hyperplane conjecture, is whether it is universally bounded (independently of $f$ and $d$ ). The best known bound is $L_{f} \leq c d^{1 / 4}$ for some universal constant $c>0$ ([19]). See [2, 20, 19, 13] for background, equivalent formulations and related results.

Theorem 6. Let $X$ be an isotropic log-concave random vector in $\mathbb{R}^{d}$. Assume that it satisfies an entropy jump with constant $\kappa \in(0,1)$ :

$$
\operatorname{Ent}\left(\frac{X+Y}{\sqrt{2}}\right)-\operatorname{Ent}(X) \geq \kappa(\operatorname{Ent}(G)-\operatorname{Ent}(X)) .
$$

for $Y$ an independent copy of $X$. By Theorem 1, this holds with $\kappa=c / 8$ if $f$ satisfies a spectral gap inequality (1.2) with constant $c>0$. Then we have

$$
L_{X} \leq e^{2 / \kappa} .
$$


Note that the bound also reads as

$$
L_{X} \leq e^{16 \mathrm{c}_{\mathrm{p}}(X)}
$$

in terms of $\mathrm{c}_{\mathrm{p}}(X):=\mathrm{c}_{\mathrm{p}}(f)$, the Poincaré constant (1.3) of $X \sim f$.

As a consequence, we see that the KLS conjecture (asserting that isotropic log-concave distributions satisfy a Poincaré inequality (1.2) with some universal constant) implies the Hyperplance conjecture. In this direction, a better result is known; indeed, Eldan and Klartag [13] recently proved that the variance conjecture implies as well the Hyperplane conjecture. The variance conjecture asserts that inequality (1.2) for the particular function $s(x)=|x|^{2}-\int|y|^{2} f(y) d y$ holds with a universal constant for every log-concave isotropic distribution $f$ on every dimension $d$. However, it is worth noting that unlike the EldanKlartag result, our estimate above holds at the level of an individual distribution $X$.

Theorem [6 was presented by the first named author in 2003 at a conference in Kiel and then expanded in a series of lectures in 2006 at the conference Phenomena in High Dimensions at the I.H.P., as part of a more general program proposing a probabilistic viewpoint on the geometry of convex bodies in high dimensions. A similar program was also recently and independently proposed by Bobkov and Madiman (see e.g. [8, 9]).

Let us now explain the short and simple argument allowing us to pass from the entropy jump to a bound on the isotropic constant. It relies on a classical rigidity property of isotropic log-concave distributions $X \sim f$ in $\mathbb{R}^{d}$, namely that up to non-essential linear terms in $d$, we have $\log f(0) \simeq-\operatorname{Ent}(X)$. The following bound

$$
-\log f(0) \leq \operatorname{Ent}(X) \leq-\log f(0)+d
$$

is for instance implicit in [15] and the easy proof is as follows. Write $f=e^{-\varphi}$ with $\varphi$ convex. For the lower bound use that $\int_{\mathbb{R}^{d}} x f(x) d x=0$ together with Jensen's inequality to get

$$
-\log f(0)=\varphi(0) \leq \int_{\mathbb{R}^{d}} \varphi(x) f(x) d x=\operatorname{Ent}(X) .
$$

The upper bound combines the convexity of $f$ and an integration by parts as follows:

$$
\operatorname{Ent}(X)-\varphi(0)=\int_{\mathbb{R}^{d}} f(x)(\varphi(x)-\varphi(0)) d x \leq \int_{\mathbb{R}^{d}} f(x) \nabla \varphi(x) \cdot x d x=d .
$$

Let us mention that in the definition of $L_{X}$ and in the entropic bounds above, we can replace, up to numerical constants, $f(0)$ by ||$f \|_{\infty}:=\sup _{\mathbb{R}^{d}}|f|$, since it is known (see [14]) that $\|f\|_{\infty} \leq e^{d} f(0)$ for an isotropic log-concave distribution $f$ on $\mathbb{R}^{d}$.

To finish the proof of (5.1), assume first that $X \sim f$ is symmetric, which means that $f$ is even. If $h$ denotes the density of $\frac{X+Y}{\sqrt{2}}$, then $h(x)=2^{\frac{d}{2}} \int_{\mathbb{R}^{d}} f(x-y) f(y) d y$. It then follows from the log-concavity of $f$ that

$$
h(0)=2^{\frac{d}{2}} \int_{\mathbb{R}^{d}} f(y)^{2} d y \geq 2^{\frac{d}{2}} \int_{\mathbb{R}^{d}} f(2 y) f(0) d y=2^{-\frac{d}{2}} f(0) .
$$


Hence, using (5.2) we have that

$$
\operatorname{Ent}\left(\frac{X+Y}{\sqrt{2}}\right) \leq \frac{d}{2} \log 2-\log f(0)+d \leq-\log f(0)+\frac{3}{2} d .
$$

Let us now go back to the general case where $X \sim f$ is not necessarily symmetric, and consider $Y, X^{\prime}, Y^{\prime}$ i.i.d. copies of $X$. Then $\frac{X-X^{\prime}}{\sqrt{2}}, \frac{Y-Y^{\prime}}{\sqrt{2}}$ are symmetric log-concave isotropic random vectors in $\mathbb{R}^{d}$, independent and identically distributed according to the density $g(x)=2^{d / 2} \int_{\mathbb{R}^{d}} f(x+y) f(y)$. It follows from the argument above that $g(0) \geq 2^{-d / 2} f(0)$. Thus, by the Shannon-Stam inequality $\frac{1}{2} \operatorname{Ent}(Z)+\frac{1}{2} \operatorname{Ent}(U) \leq \operatorname{Ent}\left(\frac{Z+U}{\sqrt{2}}\right)$ for $Z=(X+$ $Y) / \sqrt{2}$ and $U=-\left(X^{\prime}+Y^{\prime}\right) / \sqrt{2}$ two independent random vectors, and the bound (5.3) obtained in the symmetric case, one gets

$$
\begin{aligned}
\operatorname{Ent}\left(\frac{X+Y}{\sqrt{2}}\right) & \leq \operatorname{Ent}\left(\frac{X+Y-X^{\prime}-Y^{\prime}}{\sqrt{4}}\right)=\operatorname{Ent}\left(\frac{\frac{X-X^{\prime}}{\sqrt{2}}+\frac{Y-Y^{\prime}}{\sqrt{2}}}{\sqrt{2}}\right) \\
& \leq-\log g(0)+\frac{3}{2} d \\
& \leq-\log f(0)+2 d
\end{aligned}
$$

On the other hand, the assumption on the Entropy jump implies

$$
(1-\kappa) \operatorname{Ent}(X) \leq \operatorname{Ent}\left(\frac{X+Y}{\sqrt{2}}\right)-\kappa \operatorname{Ent}(G) \leq \operatorname{Ent}\left(\frac{X+Y}{\sqrt{2}}\right)
$$

since $\operatorname{Ent}(G)=\frac{d}{2} \log 2 \pi e \geq 0$. Therefore, using again (5.2) we get that

$$
(1-\kappa)(-\log f(0)) \leq-\log f(0)+2 d .
$$

This implies

$$
\kappa \log f(0) \leq 2 d
$$

and the desired bound (5.1).

\section{Acknowledgements}

The second author would like to thank his PhD advisor Dario Cordero-Erausquin for all his help and advice.

\section{References}

[1] D. Bakry and M. Émery , Diffusions hypercontractives, in Séminaire de probabilités, XIX, 1984/84, ed. J. Azéma and M. Yor, Lecture Notes in Math. 1123, Spinger, Berlin, 1985, 177-206. 
[2] K. Ball, Logarithmically concave functions and sections of convex sets in $R^{n}$, Studia Math. 88 (1988), 69-84.

[3] K. Ball, F. Barthe and A. Naor, Entropy jumps in the presence of a spectral gap, Duke Math. J. 119 (2003), 41-63.

[4] A. R. Barron, Entropy and the central limit theorem, Ann. Probab. 14 (1986), 336342.

[5] A. R. Barron, O. Johnson, Fisher information inequalities and the central limit theorem, Probab. Theory Relat. Fields. 129 (2004) 391-409.

[6] N. M. Blachman, The convolution inequality for entropy powers, IEEE Trans. Inform. Theory 2 (1965), 267-271.

[7] S. G. Bobkov, Isoperimetric and analytic inequalities for log-concave probability measures, Ann. Probab. 27 (1999), 1903-1921.

[8] S. G. Bobkov and M. Madiman, Dimensional behaviour of entropy and information, C. R. Math. Acad. Sci. Paris 349 ( 2011 ), 201-204.

[9] S. G. Bobkov and M. Madiman, The entropy per coordinate of a random vector is highly constrained under convexity conditions, IEEE Trans. Inform. Theory $5 \mathbf{7}$ (2011 ), 4940-4954.

[10] E. A. Carlen, Superadditivity of Fisher's information and logarithmic Sobolev inequalities, J. Funct. Anal. 101 1991, 194-211.

[11] E. A. Carlen and A. Soffer, Entropy production by block variable summation and central limit theorems, Comm. Math. Phys. 140 (1991), 339-371.

[12] I. Csiszár, Informationstheoretische Konvergenzbegriffe im Raum der Wahrscheinlichkeitsverteilungen, Magyar Tud. Akad. Mat. Kutató Int. Kozl. 7 (1962), 137-158.

[13] R. Eldan and B. Klartag, Approximately gaussian marginals and the hyperplane conjecture, Proc. of a Workshop on "Concentration, functional inequalities and isoperimetry", Comtermporary Math, 545, Amer. Math. Soc., (2011), 55-68.

[14] M. Fradelizi, Sections of convex bodies through their centroid, Arch. Math. 69 (1997), 515-522. 
[15] M. Fradelizi and M. Meyer, Increasing functions and inverse Santaló inequality for unconditional functions, Positivity 12 (2008), 407-420.

[16] L. Gross, Logarithmic Sobolev Inequalities, Amer. J. Math. 97 (1975), 1061-1083.

[17] M. S. Pinsker, Information and Information Stability of Random Variables and Processes, Holden-Day, San Francisco, 1964.

[18] R. Kannan, L. Lovasz, and M. Simonovits, Isoperimetric problems for convex bodies and a localization lemma, Discrete Comput. Geom. 13 (1995), 541-559.

[19] B. Klartag, On convex perturbations with a bounded isotropic constant, Geom. and Funct. Anal. 16 (2006), 1274-1290.

[20] V. Milman and A. Pajor, Isotropic position and inertia ellipsoids and zonoids of the unit ball of a normed $n$-dimensional space, in Geometric aspects of functional analysis (1987-88), Lecture Notes in Math., Vol. 1376, Springer, Berlin, (1989), 64-104.

[21] A. Prékopa, A. On logarithmic concave measures and functions, Acad Sci Math (Szeged) 34 (1973), 335-343.

[22] C. E. Shannon and W. Weaver, The Mathematical Theory of Communication, University of Illinois Press, Urbana, 1949.

[23] A. J. Stam, Some inequalities satisfied by the quantities of information of Fisher and Shannon, Inform. and Control 2 (1959), 101-112. 